\title{
Nonlinear Static Procedure for Fragility Curve Development
}

\author{
By Masanobu Shinozuka, ${ }^{1}$ Honorary Member, ASCE, \\ Maria Q. Feng, ${ }^{2}$ Associate Member, ASCE, Ho-Kyung Kim, ${ }^{3}$ and Sang-Hoon Kim ${ }^{4}$
}

\begin{abstract}
This study examines the fragility curves of a bridge by two different analytical approaches; one utilizes the time-history analysis and the other uses the capacity spectrum method. The latter approach is one of the simplified nonlinear static procedures recently developed for buildings. In this respect, a sample of 10 nominally identical but statistically different bridges and 80 ground-motion time histories are considered to account for the uncertainties related to the structural capacity and ground motion, respectively. The comparison of fragility curves by the nonlinear static procedure with those by time-history analysis indicates that the agreement is excellent for the state of at least minor damage, but not as good for the state of major damage where nonlinear effects clearly play a crucial role. Overall, however, the agreement is adequate even in the state of major damage considering the large number of typical assumptions under which the analyses of fragility characteristics are performed.
\end{abstract}

\section{INTRODUCTION}

In performing a seismic risk analysis of a structural system, it is important to identify the seismic vulnerability of component structures associated with various states of damage. It is a widely practiced approach to develop vulnerability information in the form of fragility curves. The first writer has presented the development of empirical and analytical fragility curves for bridges (Shinozuka et al. 2000). Empirical curves were developed by utilizing the damage data associated with past earthquakes, while analytical curves were developed by numerically simulating seismic response with the aid of structural dynamic analysis. Although the most reliable analytical method is the use of complete nonlinear time-history analysis, the present state of the physical understanding of the physics of damage, in general, appears to be premature for the sophistication such a rigorous analysis represents.

Recently, there has been an increasing interest in the simplified nonlinear analysis method, referred to generally as the nonlinear static procedure. A report by the Applied Technology Council (ATC) (1996) stated: “Available nonlinear static procedures include (1) the capacity spectrum method (CSM) (ATC 1996) that uses the intersection of the capacity (or pushover) curve and a reduced response spectrum to estimate maximum displacement; (2) the displacement coefficient method (FEMA 1997) that uses pushover analysis and a modified version of the equal displacement approximation to estimate maximum displacement; and (3) the secant method [e.g., City of Los Angeles (COLA 1995)] that uses a substitute structure and secant stiffness." These methods are basically targeted to the seismic evaluation and retrofit of buildings, not bridges. However, similar concepts and procedures are currently under investigation by bridge researchers and engineers to introduce

${ }^{1}$ Fred Champion Prof., Dept. of Civ. and Envir. Engrg., Univ. of Southern California, Los Angeles, CA 90089-2531.

${ }^{2}$ Assoc. Prof., Dept. of Civ. and Envir. Engrg., Univ. of California, Irvine, CA 92697-2175.

${ }^{3}$ Asst. Prof., Dept. of Civ. Engrg., Mokpo Nat. Univ., Muangun, Cheonnam 534-729, Korea; presently, Visiting Scholar, Dept. of Civ. and Envir. Engrg., Univ. of Southern California, Los Angeles, CA. CA.

${ }^{4}$ Res. Asst., Dept. of Civ. and Envir. Engrg., Univ. of California, Irvine,

Note. Associate Editor: Ahsan Kareem. Discussion open until May 1, 2001. To extend the closing date one month, a written request must be filed with the ASCE Manager of Journals. The manuscript for this paper was submitted for review and possible publication on April 6, 2000. This paper is part of the Journal of Engineering Mechanics, Vol. 126, No. 12, December, 2000. (C)ASCE, ISSN 0733-9399/00/0012-1287-1295/ $\$ 8.00+\$ .50$ per page. Paper No. 22297. standardized, simplified procedures for performance-based seismic evaluation of bridges (Barron 1999; Dutta 1999).

Conforming to this current trend, this study considers a feasible means for developing fragility curves of bridges by utilizing the CSM. Preliminarily, responses by the CSM for a set of ground-motion time histories are evaluated and compared with those by the nonlinear time-history analysis. Based on this examination, fragility curves for a typical bridge are developed by the CSM and are compared with those by the timehistory analysis, as proposed in a preceding study (Shinozuka et al. 2000).

\section{MEMPHIS BRIDGE AND SEISMIC GROUND MOTION}

To ensure the most efficient comparison between the CSM and the time-history analysis method, the same representative bridge in Memphis, with a precast, prestressed-continuous deck, and the same set of 80 time histories of ground motion as adopted in Shinozuka et al. (2000) are considered again. This bridge and the 80 time histories were originally studied by Jernigan and Hwang (1997) and Hwang and Huo (1996).

\section{Memphis Bridge}

The plan, elevation, and column cross section of the Memphis bridge are depicted in Fig. 1. Each pier has 2 columns $4.58 \mathrm{~m}$ in height. Following Jernigan and Hwang (1997), the strength $f_{c}$ of $20.7-\mathrm{MPa}$ concrete used for the bridge is assumed to be best described by a normal distribution with a mean strength of $31.0 \mathrm{MPa}$ and a standard deviation of 6.2 $\mathrm{MPa}$. The yield strength $f_{y}$ of Grade 40 reinforcing bars used in the design is described by a lognormal distribution having a mean strength of $336.2 \mathrm{MPa}$, with a standard deviation of 36.0 MPa. The same sample of 10 "nominally identical but statistically different" bridges, as used in the simulation analysis performed in Shinozuka et al. (2000), is then created for the bridge by simulating 10 realizations of $f_{c}$ and $f_{y}$ according to assumed respective probability distribution functions. These bridges are referred to as Sample Bridge 1, 2, . , 10, distinguishing them from the representative Memphis bridge. Other parameters that could contribute to the variability of the structural response were not considered in the present analysis, with the assumption that their contributions could be disregarded.

\section{Seismic Ground Motion}

For the seismic ground motion, the time histories generated by Hwang and Huo (1996) at the Center for Earthquake Re- 


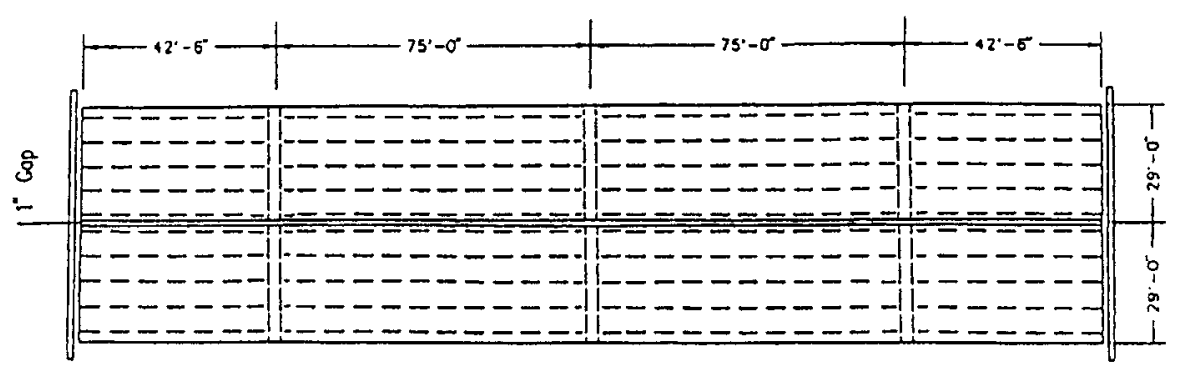

Plan
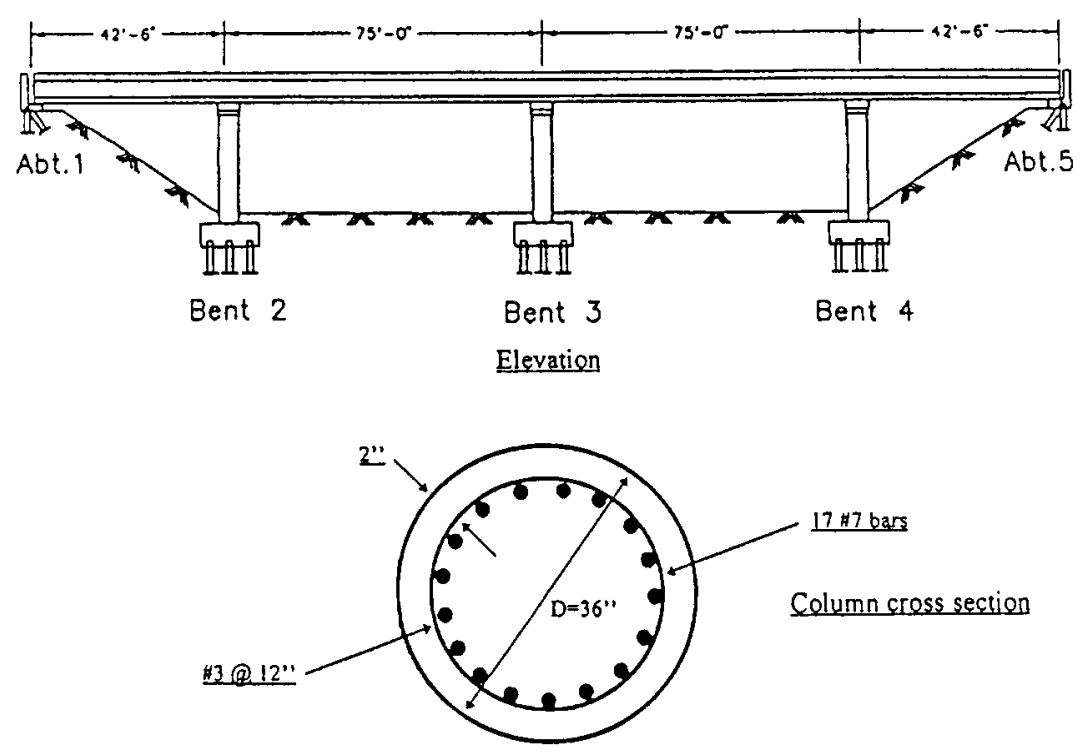

FIG. 1. Representative Memphis Bridge

search and Information at the University of Memphis are used. To minimize computational effort, samples of 10 time histories were randomly selected from 50 histories generated by Hwang and Huo (1996) for each of the following eight combinations of $M$ (magnitude) and $R$ (epicentral distance): $M=6.5$ with $R$ $=80$ and $100 \mathrm{~km}, M=7.0$ with $R=60$ and $80 \mathrm{~km}, M=7.5$ with $R=40$ and $60 \mathrm{~km}$, and $M=8.0$ with $R=40$ and $60 \mathrm{~km}$. The spectral accelerations, averaged over 10 acceleration time histories from each combination of $M$ and $R$, are shown in Fig. 2(a) with four combinations. This figure also shows the spectral acceleration averaged over 80 total acceleration time histories, to provide insight to the frequency content of these ground motion time histories. Figs. 2(b and c) show the velocity response spectrum and the displacement response spectrum, respectively, averaged over 80 total ground motions.

\section{CSM}

Two key elements of the CSM are "demand" and "capacity." Demand represents the intensity of the seismic ground motion to which bridges are subjected, while capacity represents the bridge's ability to resist the seismic demand. The determination of three primary elements are required by the CSM: Capacity spectrum, demand spectrum, and performance point. Each of these elements utilized in this study basically conforms to the report by the ATC-40 (1996), and is briefly described below using the Memphis bridge and the set of seismic ground-motion time histories introduced above as examples.

\section{Capacity Spectrum}

To determine a capacity behind the elastic limits, the pushover analysis is performed. The standard procedure for plotting the force-displacement curve is by tracking the total shear force at the column bottoms as a function of displacement of the superstructure. The lateral forces are applied in proportion to the fundamental mode shape as

$$
F_{i}=\left(w_{i} \phi_{i} / \sum_{i=1}^{N} w_{i} \phi_{i}\right) V
$$

where $F_{i}=$ lateral force of node $i(i=1,2, \ldots, N) ; w_{i}=$ dead weight assigned to node $i ; \phi_{i}=$ amplitude of the fundamental mode at node $i ; V=$ base shear; and $N=$ number of nodes (ATC 1996).

The initial fundamental mode shape can be obtained from eigenvalue analysis performed by most general-purpose structural analysis computer codes. By gradually increasing lateral forces, plastic hinges are formed in succession at the column bottoms, and the overall pushover curve deviates from the initial straight line for elastic deformation. The effective stiffness gradually decreases, and consequently, the fundamental natural period of the bridge at any state, related to the secant slope of the radial line between the origin and corresponding point on the capacity curve, gradually increases. As a result, the fundamental mode shape eventually changes significantly, and the lateral forces in the inelastic range distribute somewhat different from those in the elastic range. With very few exceptions, general-purpose nonlinear structural analysis computer codes are capable of performing eigenvalue analysis only with tangential stiffness being at any state of deformation. However, they are incapable of obtaining mode shapes and natural periods with secant stiffness, which considers gradual decrease of stiffness with inelastic deformation of plastic hinges, as in the problem under consideration. For this reason, the period 

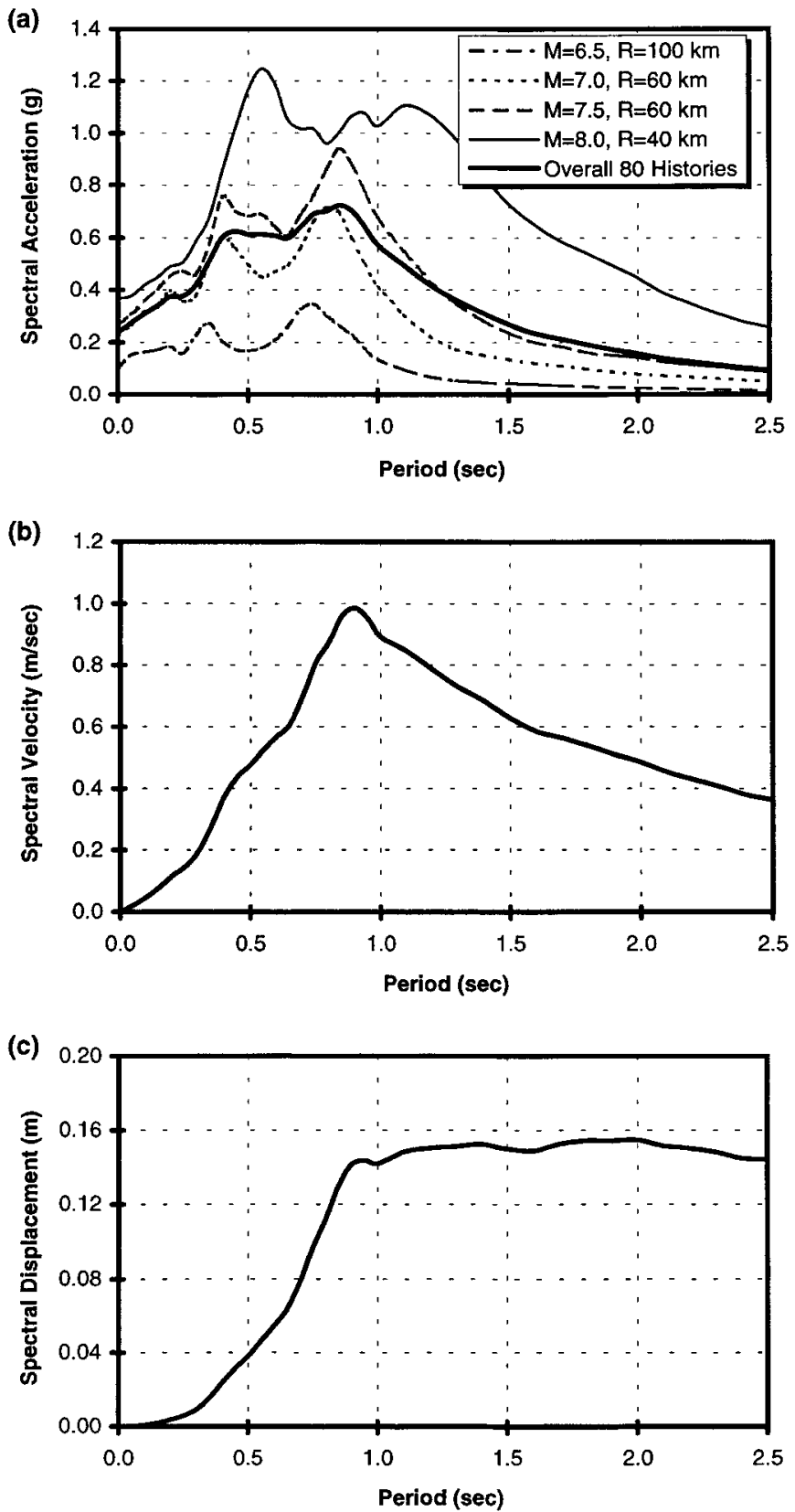

FIG. 2. Response Spectra Averaged over 80 Time Histories (5\% Damping): (a) Acceleration Response Spectra; (b) Velocity Response Spectrum; (c) Displacement Response Spectrum

and mode shape of the fundamental mode are approximated in this study by the use of the statics principle, with iterative procedures based on the following (ATC 1996):

$$
T=2 \pi \sqrt{\left(\sum_{i=1}^{N} w_{i} \delta_{i}^{2}\right) /\left(g \sum_{i=1}^{N} F_{i} \delta_{i}\right)}
$$

where $T=$ period of the fundamental mode; $\delta_{i}=$ lateral displacement at node $i$ due to lateral forces; and $g=$ acceleration due to gravity.

To use the CSM, it is necessary to convert the capacity curve to the capacity spectrum. The capacity curve expresses overall shear force on all supports as a function of the horizontal displacement of the superstructure, whereas the capacity spectrum represents the capacity curve in the acceleration-displacement response spectra (ADRS) format. The spectral acceleration $S_{a}$ and the spectral displacement $S_{d}$ can be calculated using modal parameters at any level of load magnitude as follows (ATC 1996):

$$
\begin{gathered}
S_{a}=\frac{V / W}{\alpha} \\
S_{d}=\frac{\Delta_{\text {girder }}}{P F \phi_{\text {girder }}}=\frac{\theta_{\mathrm{pl}}}{P F \phi_{\mathrm{pl}}}
\end{gathered}
$$

where $W=$ overall dead weight of bridge; $\Delta_{\text {girder }}=$ horizontal displacement of girder; $\theta_{\mathrm{pl}}=$ rotation of plastic hinge; $\phi_{\text {girder }}$ and $\phi_{\mathrm{pl}}=$ amplitudes of the fundamental mode at the girder and plastic hinge, respectively; and $P F$ and $\alpha=$ modal participation factor and modal mass coefficient, respectively, of the fundamental mode defined as follows:

$$
\begin{gathered}
P F=\left[\frac{\sum_{i=1}^{N}\left(w_{i} / \phi_{i}\right) / g}{\sum_{i=1}^{N}\left(w_{i} \phi_{i}^{2}\right) / g}\right] \\
\alpha=\frac{\left[\sum_{i=1}^{N}\left(w_{i} \phi_{i}\right) / g\right]^{2}}{\left[\sum_{i=1}^{N} w_{i} / g\right]\left[\sum_{i=1}^{N}\left(w_{i} \phi_{i}^{2}\right) / g\right]}
\end{gathered}
$$

The spectral displacement $S_{d}$ can be obtained from any displacement component of the structure as shown in (4). For bridge structures, the horizontal displacement of the girder is the most critical displacement component for developing the capacity curve. However, the rotation of the plastic hinge is more conveniently used to develop the capacity curve when the rotational ductility demand of the plastic hinge at the column bottom is used to represent the damage states as in the present study.

The DIANA 7.1 finite-element code (DIANA 1999) is utilized for the pushover analysis of the Memphis bridge. The SAP2000 (SAP2000 1999), used for the nonlinear time-history analysis in Shinozuka et al. (2000), has a special option for pushover analysis, but only with the elastic response spectrum as recommended by the ATC-40 (1996). The SAP2000 (SAP2000 1999) also covers some local nonlinear problems in dynamics, but not those in statics; this is the primary reason for the use of another code in this study. Both finite-element models for nonlinear static pushover analysis and for nonlinear time-history analysis are conceptually the same, although they are developed for different computer codes. The bridge is modeled as a fishbone model with frame, spring, and inelastic hinge elements. The modeling of plastic hinge with inelastic hinge elements is illustrated in Fig. 3. The column is modeled as an elastic frame element of length $H_{e}$, with a plastic zone of length $L_{p}$ at the column bottom. The moment-rotation relationship of the nonlinear hinge is established using the column ductility program COLx (COLx 1993).

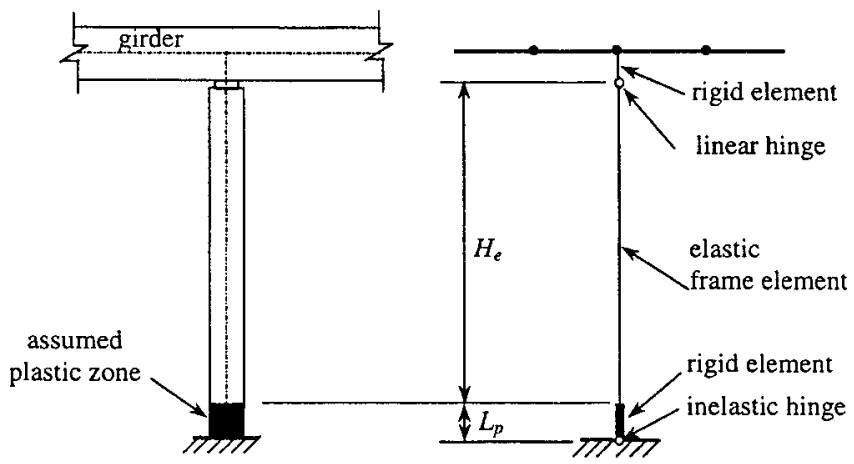

FIG. 3. Nonlinear Model of Columns 


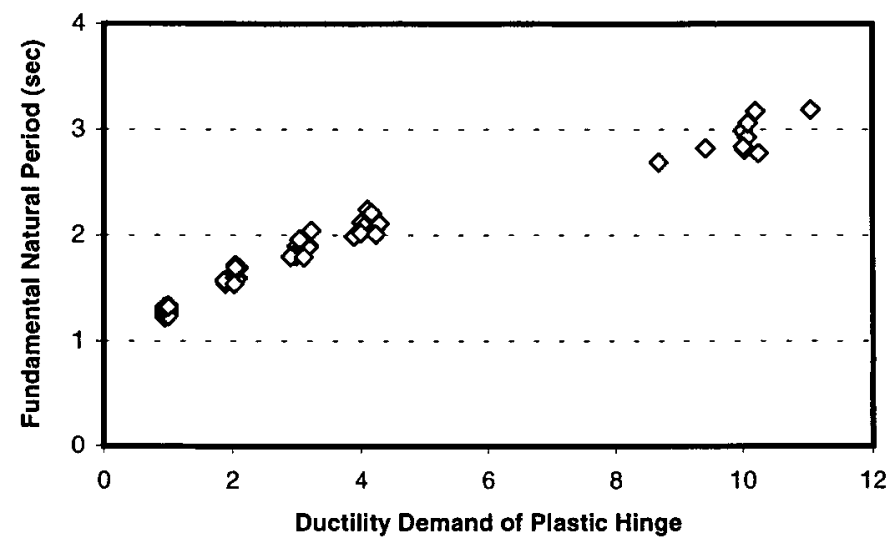

FIG. 4. Fundamental Natural Periods of 10 Sample Bridges on or near Ductility Demand of Plastic Hinge Equal to 1, 2, 3, 4, and 10

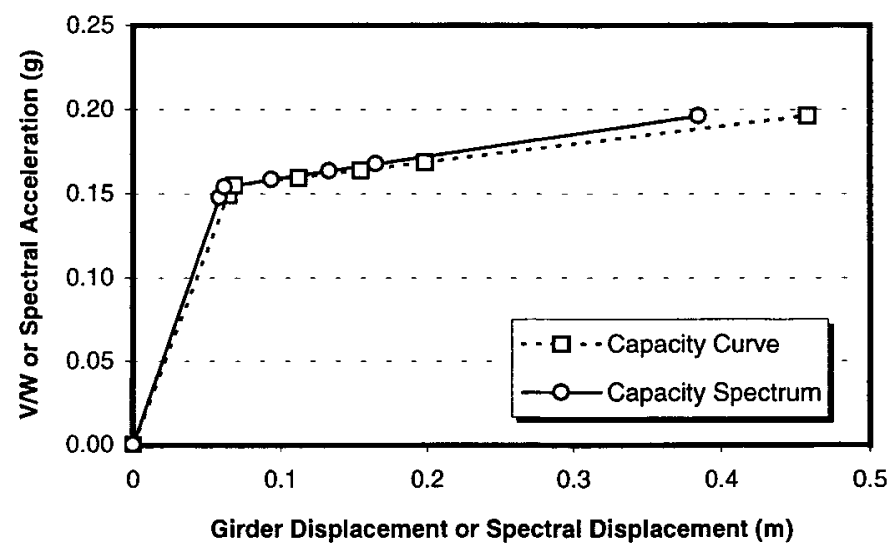

FIG. 5. Capacity Curve versus Capacity Spectrum of Sample Bridge 1

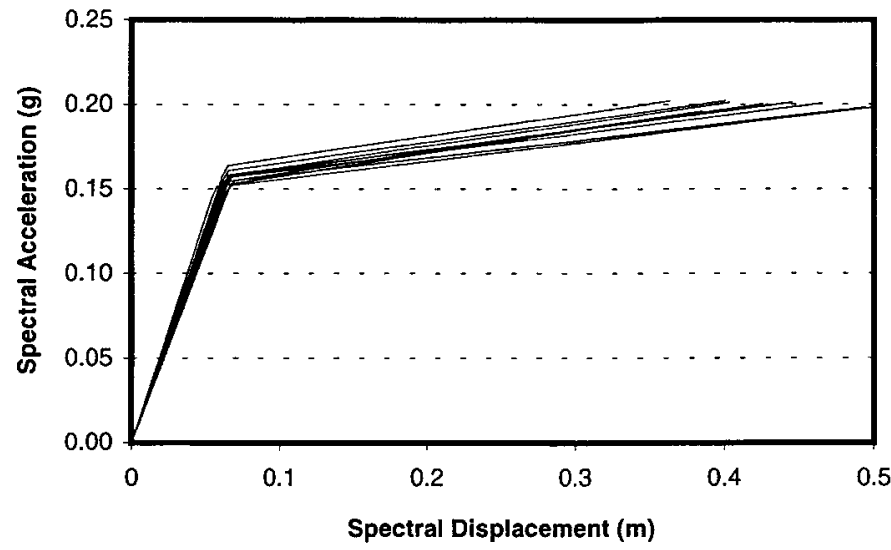

FIG. 6. Capacity Spectra of 10 Sample Bridges

Static pushover analyses are performed for 10 sample bridges to develop capacity curves. The spectral displacement in (4) is expressed with the rotation of the plastic hinge, as mentioned earlier. The bridge consists of three symmetrically positioned piers along the longitudinal axis. By increasing the lateral forces, it is found that the internal pier yields first and the external piers yield later. This slightly delayed yielding of external piers results in a smaller rotation of plastic hinges of external piers. To ensure the consistency in evaluating the minimum ductility demand of all columns at performance point, the rotation of the external pier is taken as $\phi_{\mathrm{pl}}$ in (4). The modal parameters in (3) and (4) gradually change while the plastic hinges undergo rotations beyond the yielding limit. Hence, modal parameters are calculated in several loading states and are linearly interpolated between the calculated points. These modal parameters are calculated on or near the ductility demand of the plastic hinge equal to $1,2,3,4$, and 10. Fundamental natural periods of 10 bridges at those ductility demands are calculated using (2) and are presented in Fig. 4. As shown in this figure, the fundamental natural periods for 10 bridges fall into the range from approximately 1.2 to 3.0 s. Fig. 5 shows the capacity curve of Sample Bridge 1 with the corresponding transformed capacity spectrum for the aforementioned ductility demands. Finally, Fig. 6 shows capacity spectra for the 10 sample bridges.

\section{Demand Spectrum}

The standard elastic acceleration response spectrum can be converted to ADRS format with the aid of the following (ATC 1996):

$$
S_{d}=\frac{T^{2}}{4 \pi^{2}} S_{a} g
$$

According to the ATC (1996), the reduced inelastic ADRS is developed by multiplying the reduction factors $S R_{A}$ and $S R_{V}$ for the range of constant spectral peak acceleration and constant spectral peak velocity, respectively, as follows:

$$
\begin{aligned}
& S R_{A}=\frac{3.21-0.68 \ln \left(\beta_{\text {eff }}\right)}{2.12} \geq \text { values in Table } 1 \\
& S R_{V}=\frac{2.31-0.41 \ln \left(\beta_{\text {eff }}\right)}{1.65} \geq \text { values in Table } 1
\end{aligned}
$$

where $\beta_{\text {eff }}$ (in percent) = effective viscous damping including assumed 5\% structural viscous damping as follows:

$$
\beta_{\text {eff }}=\kappa \beta_{0}+5=\frac{63.7 \kappa\left(a_{y} d_{p i}-d_{y} a_{p i}\right)}{a_{p i} d_{p i}}+5
$$

where $\beta_{0}=$ equivalent viscous damping associated with full hysteresis loop area of capacity spectrum; and $\kappa=$ damping modification factor to compensate for the uncertainty in $\beta_{0}$ because of probable imperfections in real bridge hysteresis loops, and defined as a function of structural behavior type, as shown in Table 2. Also in (10), $d_{y}, a_{y}$ represents the yielding points on the bilinear capacity spectrum, and $d_{p i}, a_{p i}$ are the performance points on the bilinear capacity spectrum at the $i$ th trial with the significance of yield points and performance points graphically depicted later in Fig. 10.

\section{Performance Point}

When the displacement $d_{p i}$ at the intersection of the reduced demand spectrum with the capacity spectrum falls within the

TABLE 1. Minimum Allowable $S R_{A}$ and $S R_{V}$ Values (ATC 1996)

\begin{tabular}{c|c|c}
\hline \hline $\begin{array}{c}\text { Structural behavior type } \\
(1)\end{array}$ & $\begin{array}{c}S R_{A} \\
(2)\end{array}$ & $\begin{array}{c}S R_{V} \\
(3)\end{array}$ \\
\hline A & 0.33 & 0.50 \\
B & 0.44 & 0.56 \\
C & 0.56 & 0.67 \\
\hline \hline
\end{tabular}

TABLE 2. Values for Damping Modification Factor $\kappa$ (ATC) 1996)

\begin{tabular}{c|c|c}
\hline \hline $\begin{array}{c}\text { Structural behavior } \\
\text { type }\end{array}$ & $\beta_{0}$ & $\kappa$ \\
$(1)$ & $(2)$ & $(3)$ \\
\hline $\mathrm{A}$ & $\leq 16.25$ & 1.0 \\
& $>16.25$ & $1.13-0.008 \beta_{0} \geq 0.77$ \\
$\mathrm{~B}$ & $\leq 25$ & 0.67 \\
& $>25$ & $0.845-0.007 \beta_{0} \geq 0.53$ \\
$\mathrm{C}$ & Any value & 0.33 \\
\hline \hline
\end{tabular}


$\pm 5 \%$ range of the displacement of the performance point obtained at the $i-1$ th iteration [i.e., $0.95 d_{p(i-1)} \leq d_{p i} \leq$ $\left.1.05 d_{p(i-1)}\right], d_{p i}$ becomes the performance point. If the intersection of the reduced demand spectrum and the capacity spectrum is not within the acceptable tolerance, then the iterative process will proceed. Basically, the ATC (1996) suggested three different procedures that standardize and simplify this iterative process (Procedures A, B, and C). These alternate procedures are based on the same concepts and mathematical relationships but vary in their dependence on analytical versus graphical methods. This study utilizes Procedure A, which is more of an analytical method than a graphical method.

\section{SINGLE GROUND-MOTION EXCITATION}

To examine more clearly the differences between evaluated responses, two methods - the CSM and the time-history analysis method - responses are calculated and compared for a set of single ground-motion time histories prior to comparing fragility curves. For this purpose, Sample Bridge 1 and eight ground-motion time histories are selected. The eight groundmotion time histories represent a randomly chosen one-time history for each combination of $M$ and $R$ (eight total combinations) to cover the wide range of peak ground acceleration (PGA).

While time-history analysis gives a unique response for each ground-motion time history, the response by the CSM usually depends on the engineer's judgments for some parameters. One of them is the "structural behavior type" when the procedure in ATC-40 (1996) is considered. The selection of structural behavior type in ATC-40 (1996) depends on both the quality of the primary elements of the seismic resisting system and the duration of shaking. Even though ATC-40 (1996) suggested the criteria to determine an appropriate type for a given structure, it is not so clear to determine one proper category for this example of the purely idealized bridge. Based on (8)-(10), the selection of structural behavior type affects the response of the bridge in connection with two parameters, such as the "minimum allowable values of reduction factors" in Table 1 and the "damping modification factor" $\kappa$ in Table 2 . Both parameters play an important role in determining the spectral reduction factors, $S R_{A}$ and $S R_{V}$.

Considering that any probable imperfection in real bridge hysteresis loops is not considered in time-history analysis, $S R_{A}$ and $S R_{V}$ in (8) and (9) can be obtained without reducing $\beta_{0}$ with $\kappa$ (i.e., $\kappa=1.0)$ in (10) and without limiting by the minimum allowable values in Table 1 . This case (hereinafter referred to as the "no modification" case) may overestimate the effective damping, resulting in an overly reduced response. The structural behavior Type A is considered to be the most reasonable assumption in this idealized example, while the structural behavior Types B or C seems not to be so appropriate. However, the sensitivity analysis of this parameter is valuable because the reduction of the elastic response spectrum is the core of the CSM. Accordingly, four possible casescases of structural behavior Types A, B, and C and one additional case of no modification - are considered in case studies. As mentioned earlier, the fundamental natural periods are over $1.2 \mathrm{~s}$ for the bridge under consideration, and Fig. 2(c) shows that these periods belong to the range of constant-peak spectral displacement. Nevertheless, $S R_{V}$, the reduction factor for the range of constant-peak spectral velocity, is applied to obtain the reduced inelastic demand spectrum because the reduction factor for the range of constant peak spectral displacement is not defined in ATC-40 (1996).

The maximum horizontal displacement of the girder and the maximum total shear force at the column bottoms by both methods are examined in this example. The response ratios, defined as the response by the CSM divided by the response by the time-history analysis, are plotted for the corresponding PGA values of eight time histories as shown in Fig. 7. For the maximum horizontal displacement of the girder, the responses by the two methods are almost the same up to a PGA of $0.2 g$. In fact, the CSM gives slightly greater displacements than the time-history analysis for this range. The structural behavior type does not affect the calculated responses because the bridge still deforms in the elastic range. This fact can be more clearly demonstrated by plotting the same response ratios as functions of the ductility demand of the plastic hinge rather than PGA, as shown in Fig. 8. This figure shows that the ductility demands are $<1.0$ for the case up to PGA of $0.2 g$, which means the bridge deforms in the elastic range. For PGA between $0.2 \mathrm{~g}$ and $0.3 \mathrm{~g}$, the difference in the response by the two methods increases up to $20 \%$, as shown in Fig. 7(a). Fig. 8(a) also shows that the ductility demands for this case range between 1.0 and 2.0. For this case, the maximum horizontal displacement is slightly influenced by the structural behavior type. For a high range of PGA between $0.3 g$ and $0.4 g$, the possible error in predicting the maximum horizontal displacement between the two methods reaches $50 \%$ depending on the structural behavior type. Fig. 8(a) shows that the ductility demands for this range of PGA can exceed 5.0. In this case of highly inelastic deformation, the CSM overestimates the hysteretic damping, and as a result, underestimates displacement considerably. It is also found that the case of structural Type A is practically the same as the case of no modification for all ground-motion time histories considered. On the other hand, the case of structural behavior Types $B$ and $C$ give significantly increased maximum displacement compared with the case of no modification, although the maximum displacements for these cases are still less than those by the time-history analysis. The same trend is shown in Figs. 7(b) and 8(b) for
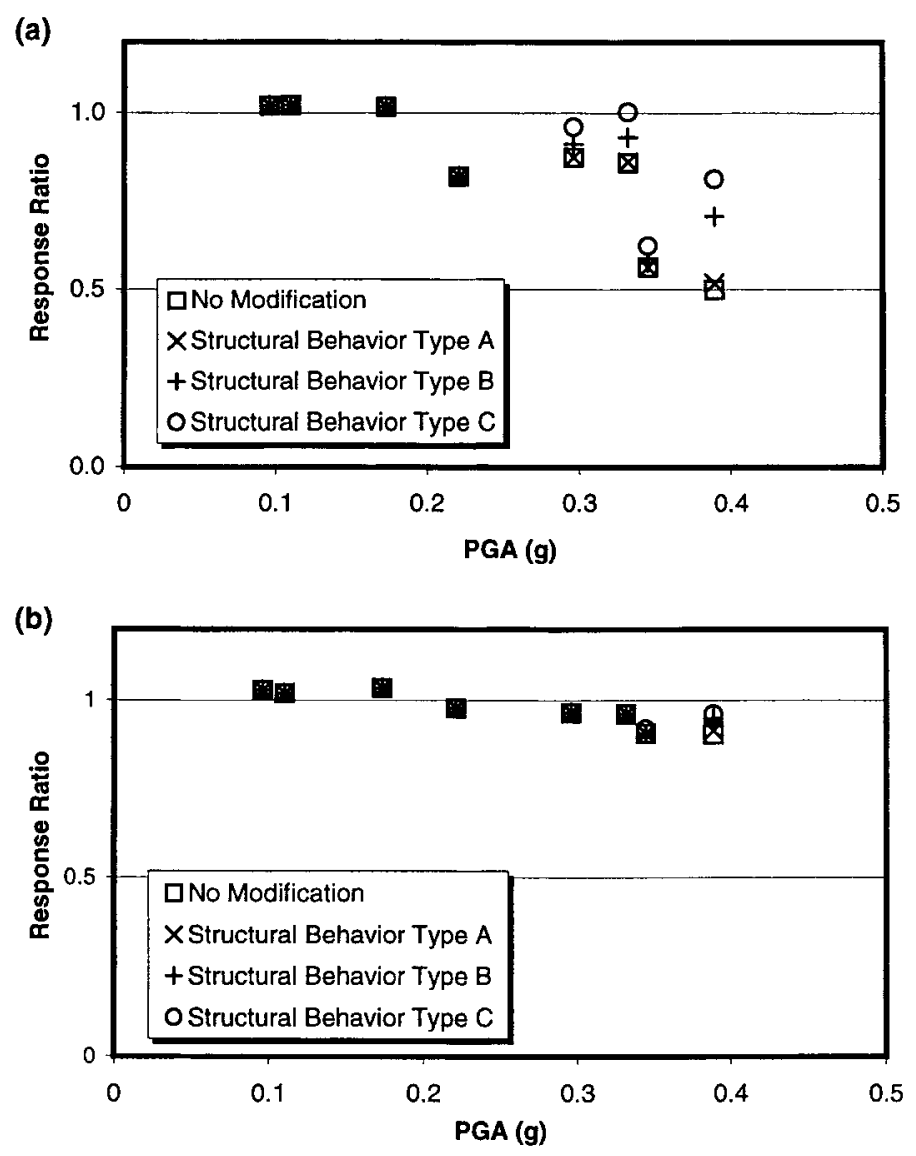

FIG. 7. Response Ratios of Sample Bridge 1 as Function of PGA: (a) Maximum Horizontal Displacement of Girder; (b) Maximum Total Shear Forces at Column Bottoms 

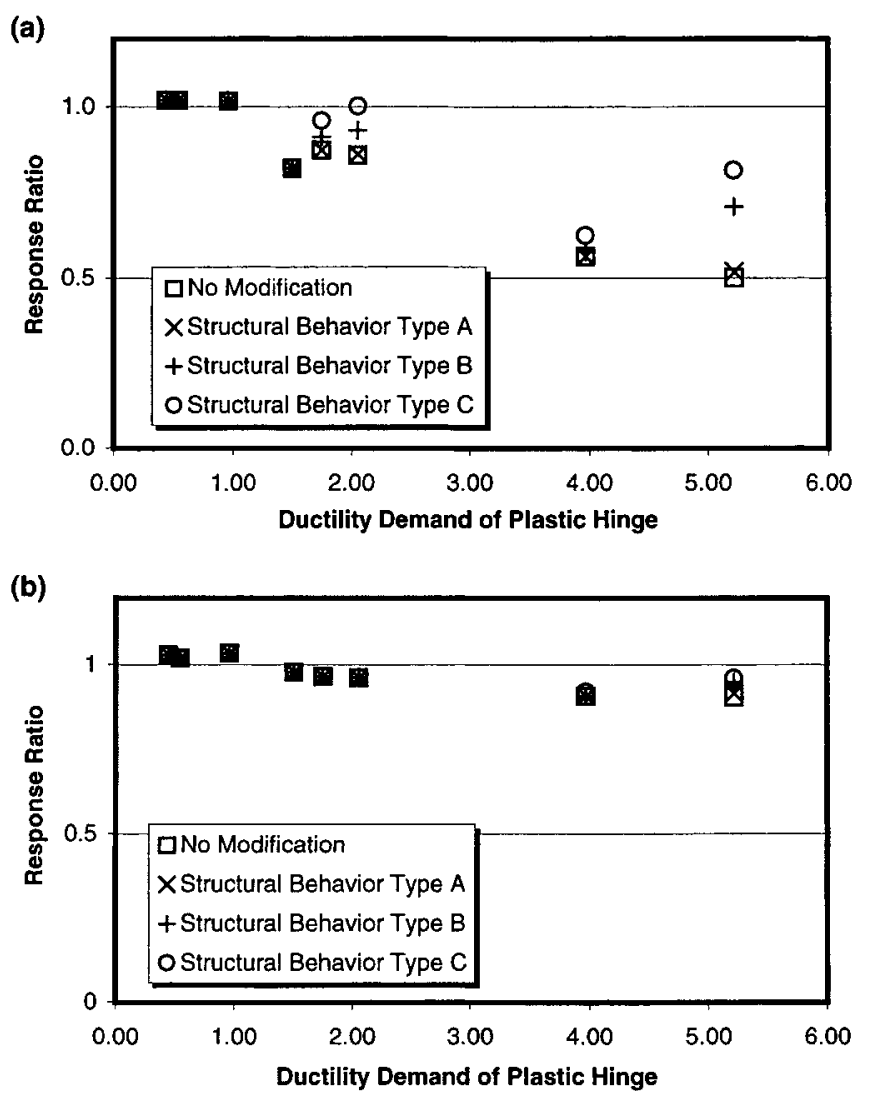

FIG. 8. Response Ratios of Sample Bridge 1 as Function of Ductility Demand of Plastic Hinge at External Pier (Ductility Demand Based on Time-History Analysis): (a) Maximum Horizontal Displacement of Girder; (b) Maximum Total Shear Forces at Column Bottoms

the maximum total shear force, where the difference between the two methods is negligible because the shear force changes relatively little after yielding.

\section{FRAGILITY CURVE}

It is assumed that fragility curves can be expressed in the form of two-parameter (median and log-standard deviation) lognormal distribution functions. For this purpose, the PGA is used to represent the intensity of the seismic ground motion even though the use of intensity measures other than PGA such as peak ground velocity, spectral acceleration, spectral intensity, and modified Mercalli intensity for fragility curve development is possible; in fact, the usefulness of these measures are examined systematically by Shinozuka et al. (2000).

\section{Fragility Curves by Time-History Analysis (Shinozuka et al. 2000)}

The estimation of the two parameters (median and log-standard deviation of the lognormal distribution) is performed with the aid of the maximum likelihood method. The likelihood function $L$ for the present purpose is expressed as

$$
L=\prod_{j=1}^{K}\left[F\left(a_{j}\right)\right]^{x_{j}}\left[1-F\left(a_{j}\right)\right]^{1-x_{j}}
$$

where $F(\cdot)$ represents the fragility curve for a specific state of damage; $a_{j}=$ PGA value to which bridge $j$ is subjected; $x_{j}=$ 1 or 0 depending on whether or not the bridge sustains the state of damage under PGA $=a_{j}$; and $K=$ total number of bridges inspected after the earthquake. Under the current lognormal assumption, $F(a)$ takes the following analytical form:

$$
F(a)=\Phi\left[\frac{\ln \left(\frac{a}{c}\right)}{\zeta}\right]
$$

where $a=\mathrm{PGA}$; and $\Phi[\cdot]$ is the standardized normal distribution function. The two parameters $c$ and $\zeta$ in (12) are computed as $c_{0}$ and $\zeta_{0}$ satisfying the following equation to maximize $\ln L$ and hence $L$ :

$$
\frac{d \ln L}{d c}=\frac{d \ln L}{d \zeta}=0
$$

This computation is performed by implementing a straightforward optimization algorithm.

\section{Fragility Curves by CSM}

The CSM is considered as a very simplified and yet as judicious a procedure as possible in evaluating bridge response using a code-type predetermined response spectrum, rather than an individual spectrum associated with a particular ground-motion time history. To enjoy the most benefit that the CSM offers, fragility curves are developed along with the following approaches.

Ground-motion time histories are sorted by PGA and grouped to the nearest representative PGA (e.g., 0.10, 0.15, $0.20, \ldots, 0.40)$ with scaling. For each group of PGA, the mean and standard deviation of the elastic acceleration response spectra for all the time histories in the group are calculated for the considered range of structural period. By developing three elastic acceleration response spectra in this way (i.e., mean and mean $\pm 1 \mathrm{SD}$ ) and transforming them to ADRS format, three ADRS [i.e., mean $(m)$ and mean $\pm 1 \mathrm{SD}(m \pm$ $\sigma)$ ] can be developed. An example of these ADRS is shown in Fig. 9 for the time histories grouped to the representative $\mathrm{PGA}=0.25 \mathrm{~g}$. A capacity spectrum for each sample bridge is then constructed one at a time, and drawn on the same coordinates. The three performance points for each of the capacity spectra are determined as its intersections with $m$ and $m \pm \sigma$ ADRS reduced properly, using the reduction factors introduced in (8) and (9). These three spectral displacements are defined as $\bar{S}_{d}(a)$ and $\bar{S}_{d}(a) \pm \sigma_{d}(a)$ and are shown in Fig. 10 for the time histories grouped to PGA $=0.25 \mathrm{~g}$. Indeed, they are functions of $a$ (PGA) since the three ADRS are developed on the time histories sorted and grouped by PGA.

$\bar{S}_{d}(a)$ and $\bar{S}_{d}(a) \pm \sigma_{d}(a)$ of the Sample Bridge 1 for each PGA are shown in Fig. 11. This figure shows that $\bar{S}_{d}(a)$, $\sigma_{d}^{+}(a)$, and $\sigma_{d}^{-}(a)$ increase gradually as PGA increases. It is also found that the magnitude of $\sigma_{d}^{+}(a)$ at any PGA is not the same as that of $\sigma_{d}^{-}(a)$. In other words, the distribution of spec-

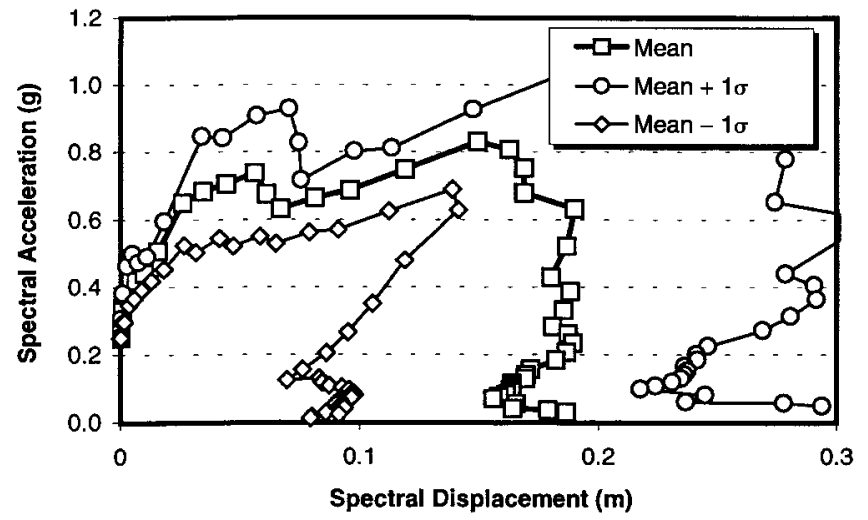

FIG. 9. Mean, Mean $+1 \sigma$ and Mean $-1 \sigma$ ADRS for Time Histories Grouped to PGA $=0.25 \mathrm{~g}$ 

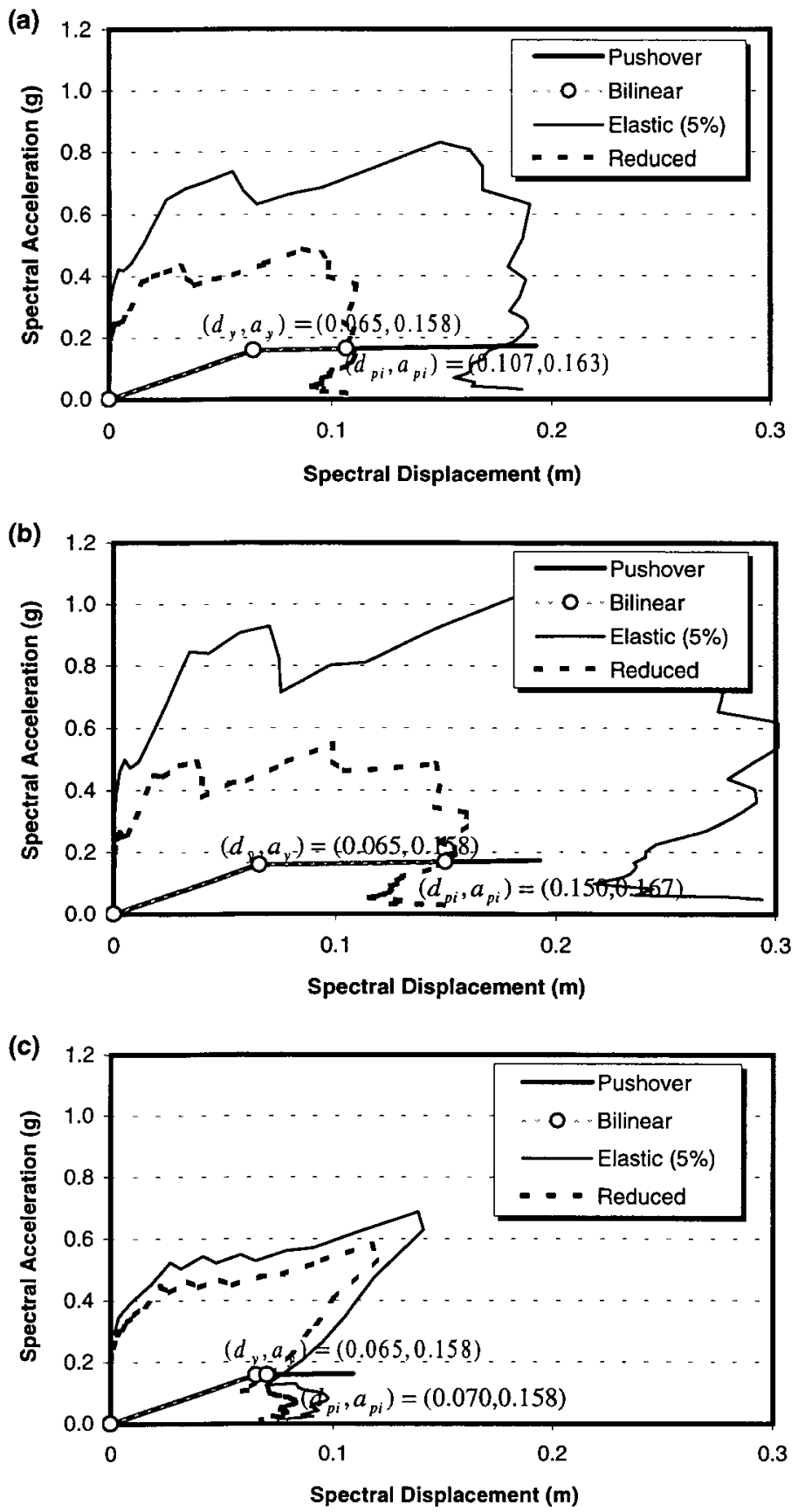

FIG. 10. Calculation of Performance Displacement for Time Histories Grouped to PGA = 0.25g: (a) Mean ADRS; (b) Mean + $1 \sigma$ ADRS; (c) Mean - 1o ADRS

tral displacement for given PGA is not symmetric. This study assumes that the spectral displacement has the mean $\bar{S}_{d}(a)$ and the standard deviation $\sigma_{d}(a)$ redefined as $\sqrt{\sigma_{d}^{+}(a) \cdot \sigma_{d}^{-}(a)}$.

Assuming the two-parameter lognormal distribution for the spectral displacement, the parameters can be obtained from the following equations:

$$
\begin{gathered}
\bar{S}_{d}(a)=c(a) \exp \left[\{\zeta(a)\}^{2} / 2\right] \\
\left\{\sigma_{d}(a)\right\}^{2}=\left\{\bar{S}_{d}(a)\right\}^{2}\left[\exp \left(\{\zeta(a)\}^{2}\right)-1\right]
\end{gathered}
$$

The limit displacement $d_{l}$ defined as the spectral displacement $S_{d}(a)$ for the specified state of damage can be expressed by (16) with the aid of (4)

$$
d_{l}=\frac{\theta_{\mathrm{pl}} / \theta_{y}}{P F \phi_{\mathrm{pl}} / \theta_{y}}=\frac{(\text { ductility demand })_{\mathrm{damage}}}{\left(P F \phi_{\mathrm{pl}}\right)_{\mathrm{damage}} / \theta_{y}}
$$

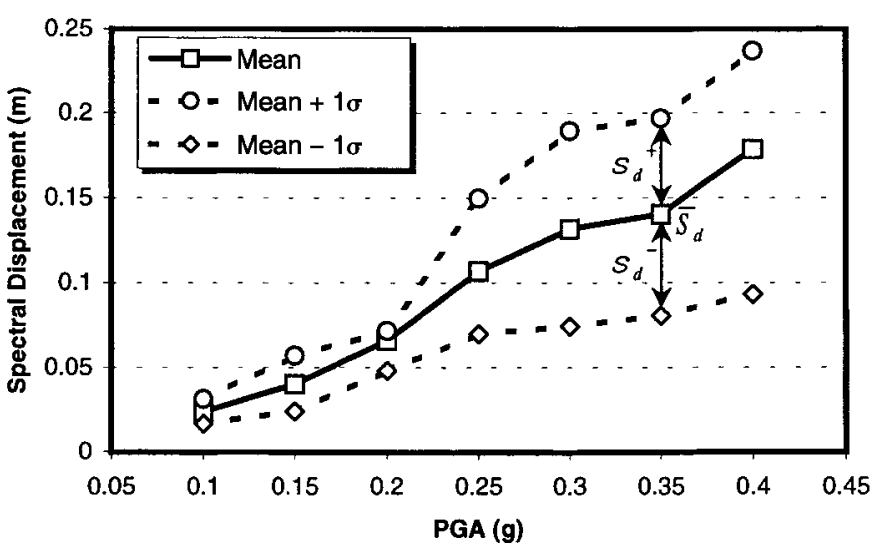

FIG. 11. Mean, Mean $+1 \sigma$, and Mean $-1 \sigma$ Performance Displacement of Sample Bridge 1

where $(X)_{\text {damage }}$ denotes the value of $X$ at the specified state of damage; and $\theta_{y}=$ yielding rotation of the plastic hinge. To be consistent with the analytical fragility curves for the Memphis bridge developed in Shinozuka et al. (2000), the state of damage by ductility demand being larger than 1.0 (minor) or 2.0 (major), exists simultaneously for all the bridge columns. Hence, (ductility demand) damage in (16) should be 1.0 for the state of at least minor damage and 2.0 for the state of major damage. It should be mentioned that the limit displacement $d_{l}$ for each sample bridge is slightly different from the other for even the same state of damage due to $\left(P F \phi_{\mathrm{pl}}\right)_{\text {damage }}$ not being identical for each sample bridge. The probability that sample bridge $j$ will have a state of damage exceeding $d_{l}$ is given by

$$
=1-\Phi\left[\frac{\ln \left(\frac{d_{l, j}}{c_{, j}(a)}\right)}{\zeta_{, j}(a)}\right]
$$$$
P\left[S_{d}(a) \geq d_{l} \text { for sample bridge } j\right]=P_{j}\left(a, d_{l}\right)
$$

where $d_{l}=\left(d_{l}\right)_{1}$ and $d_{l}=\left(d_{l}\right)_{2}$ represent, respectively, the states of minor and major damage under the assumption that the same state of damage will be imposed on all the columns simultaneously, as the nonlinear static analysis tends to imply. The subscript $j$ on $d_{l, j}, c_{, j}(a)$, and $\zeta_{, j}(a)$ in (17) explicitly denotes that these three parameters are dependent on each sample bridge $j$. The fragility value at $\mathrm{PGA}=a$ for the state of damage represented by $d_{l}$ can be estimated by taking all $K$ bridges in the PGA group under consideration as follows:

$$
F\left(a, d_{l}\right)=\frac{\sum_{j=1}^{K} P_{j}\left(a, d_{l}\right)}{K}
$$

These values are plotted in terms of open squares in Fig. 12 , whereas the values of $P_{j}\left(a, d_{l}\right)$ are plotted in terms of crosses to show the degree of fragility variation due to the variability in structural characteristics. Eighty diamonds plotted on the two horizontal axes, which represent $x_{i}=0$ and $x_{i}$ $=1$ in relation to (11), and the fragility curve by the timehistory method are replotted here from Shinozuka et al. (2000). The comparison of these results indicates that the agreement is excellent for the state of at least minor damage, but it is not as good for the state of major damage where nonlinear effects obviously play a crucial role. Overall, however, the agreement is adequate even in the case dealing with the state of major damage, considering a large number of typical assumptions under which the analysis of fragility characteristics are performed.

Fig. 13 shows the sensitivity of the structural behavior type 
(a)

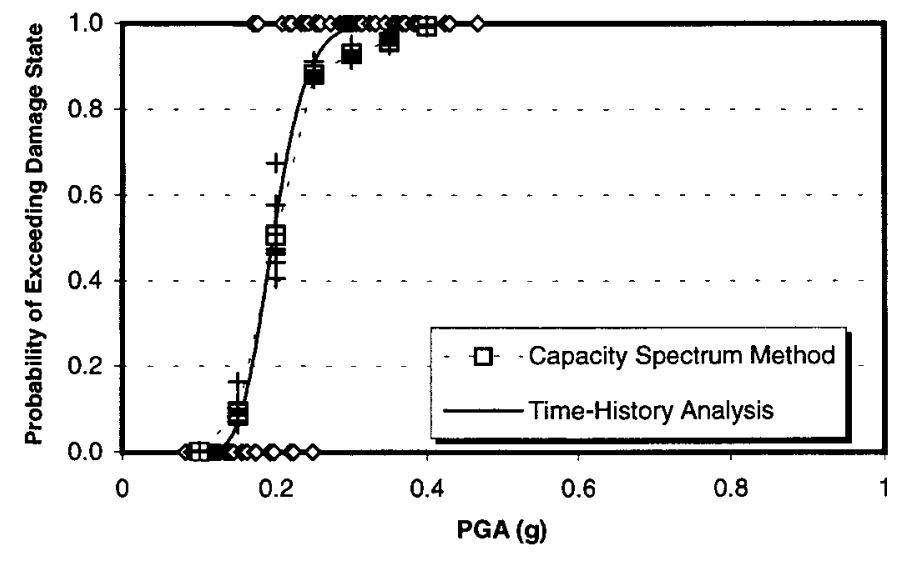

(b)

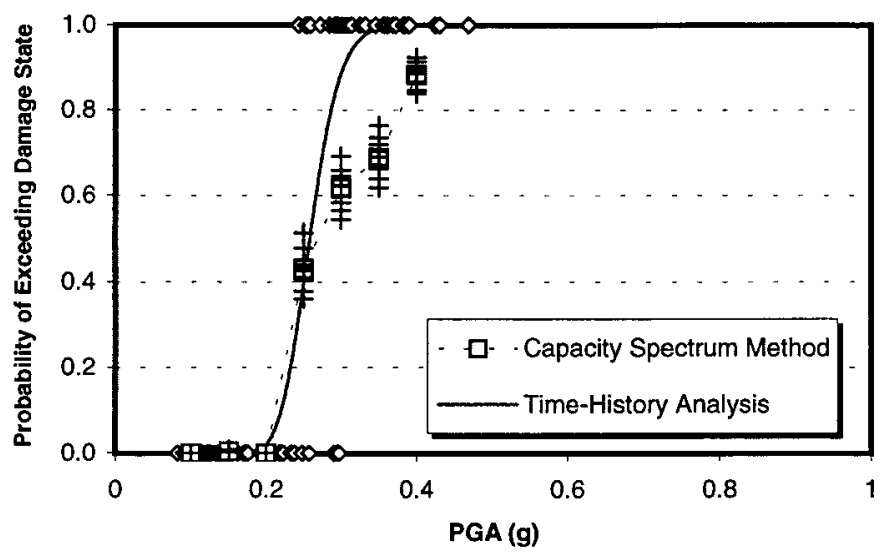

FIG. 12. Fragility Curves of 10 Sample Bridges: (a) State of at Least Minor Damage; (b) State of Major Damage

to the fragility curves. Fig. 13(a) shows that the significance of the structural behavior type can be neglected in developing the fragility curve for the state of at least minor damage. On the contrary, Fig. 13(b) shows the dependency of the fragility curve for the state of major damage on the structural behavior type.

\section{DISCUSSIONS AND CONCLUSIONS}

This study examines the fragility curves of bridges by two different analytical approaches; one utilizes the time-history analysis approach and the other makes use of the CSM. The latter is one of the simplified nonlinear static procedures recently developed, or currently in development, mainly for building structures.

The comparison of fragility curves by the nonlinear static procedure with those by the time-history analysis indicates that the agreement is excellent for the state of at least minor damage, but it is not as good for the state of major damage where nonlinear effects obviously play a crucial role. This result appears to be attributable to the following reasons. First, any possible imperfection in real bridge hysteresis loops is not considered in the finite-element models. But, the spectral reduction factors found in ATC-40 (1996) are basically determined for real structures which show considerable degradation in strength and stiffness by hysteretic cycling deformation. This could lead to the discrepancy between the two methods especially for the cases of strong ground motion, Second, the spectral reduction factors used in the case studies are for the range of constant peak spectral velocity. ATC-40 (1996) suggests the spectral reduction factors only for two ranges of constant peak spectral acceleration and constant peak spectral ve- (a)

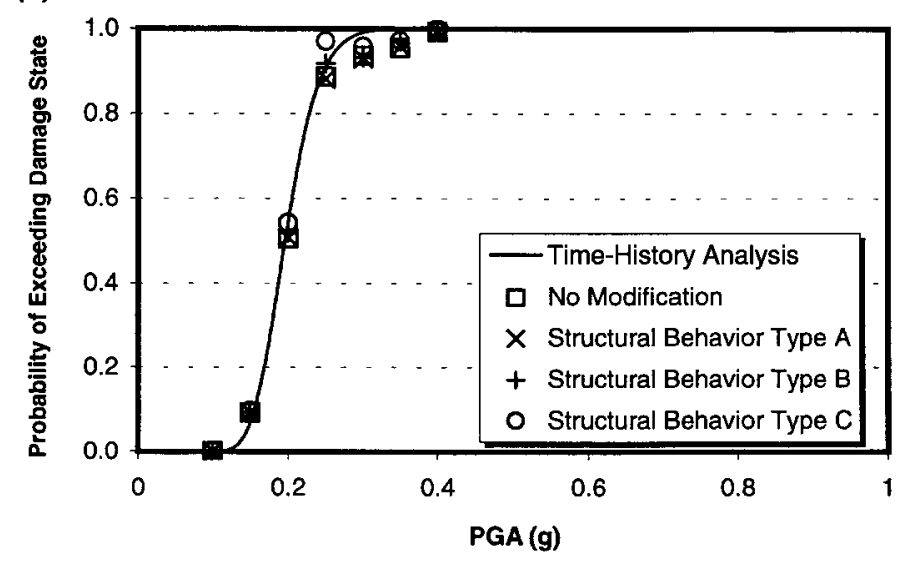

(b)

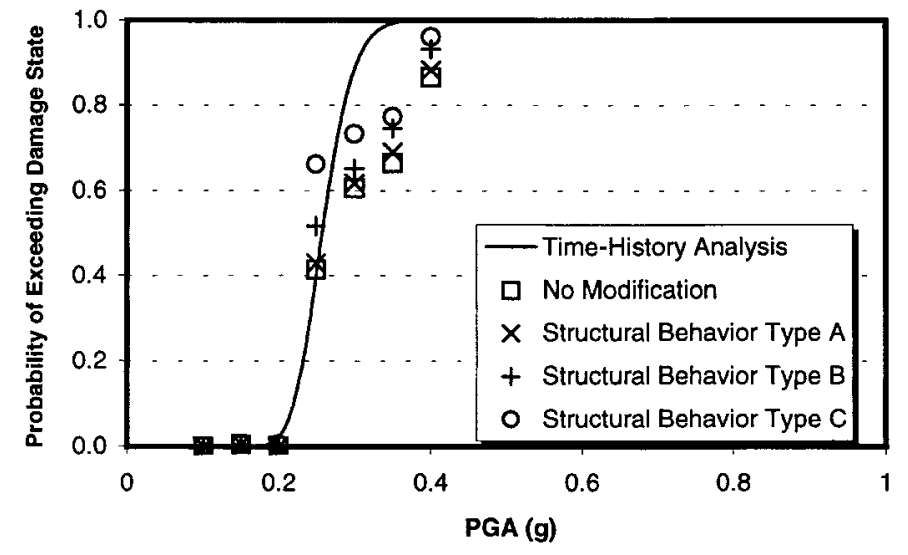

FIG. 13. Fragility Curves of Memphis Bridge: (a) State of at Least Minor Damage; (b) State of Major Damage

locity. But the response spectra of utilized ground motions consist of only two ranges of constant peak-the range of constant peak spectral acceleration and the range of constant peak spectral displacement. Considering the fundamental natural periods of the bridges under investigation, the spectral reduction factor should be taken for the range of constant peak spectral displacement rather than constant peak spectral velocity. This effect should be examined in detail by using other ground-motion time histories in future studies. Although the CSM in ATC-40 (1996) suggests a more simplified practical tool for evaluating seismic response, the theoretical background of estimating effective hysteretic damping should be validated more clearly in future studies.

Overall, however, the agreement is adequate even in the case dealing with the major state of damage considering a large number of typical assumptions under which the analyses of fragility characteristics are performed.

\section{ACKNOWLEDGMENTS}

In carrying out this work, the first and third writers were supported by the Federal Highway Administration, McLean, Va., through the Multidisciplinary Center for Earthquake Engineering Research, Buffalo, N.Y., under Contract Nos. DTFH61-92-C00112 and DTFH61-92-C-00106 and by the National Science Foundation, Arlington, Va., through the Multidisciplinary Center for Earthquake Engineering Research under Contract Nos. R90623 and R92629. The third writer was also supported by the Korea Science and Engineering Foundation, Seoul, Korea, as a postdoctoral fellow. The second and fourth writers were supported by the $\mathrm{Na}$ tional Science Foundation under Grant No. CMS-9812585.

\section{APPENDIX I. REFERENCES}

Applied Technology Council (ATC). (1996). "Seismic evaluation and retrofit of concrete buildings." Rep. No. SSC 96-01: ATC-40,1, Redwood City, Calif. 
Barron, R. (1999). "Spectral evaluation of seismic fragility of structures." PhD dissertation, Dept. of Civ., Struct. and Envir. Engrg., State University of New York at Buffalo, Buffalo.

City of Los Angeles (COLA). (1995). "Earthquake hazard reduction in existing reinforced concrete buildings and concrete frame buildings with masonry infills." Tech. Rep., Los Angeles.

COLx user's manual. (1993). California Department of Transportation (Caltrans), Sacramento, Calif.

DIANA user's manual-Release 7.1. (1999). TNO Building and Construction Research, Delft, The Netherlands.

Dutta, A. (1999). "On energy based seismic analysis and design of highway bridges." PhD dissertation, Dept. of Civ., Struct. and Envir. Engrg., State University of New York at Buffalo, Buffalo.

Federal Emergency Management Agency (FEMA). (1997). "NEHRP guidelines for the seismic rehabilitation of buildings." FEMA-273, Washington, D.C.

Hwang, H. M., and Huo, J.-R. (1996). "Simulation of earthquake acceleration time histories." Tech. Rep., Ctr. for Earthquake Res. and Information, University of Memphis, Memphis.

Jernigan, J. B., and Hwang, H. M. (1997). "Inventory and fragility analysis of Memphis bridges." Tech. Rep., Ctr. for Earthquake Res. and Information, University of Memphis, Memphis.

SAP2000 user's manual-Nonlinear version 7.1. (1999). Computers and Structures, Inc., Berkeley, Calif.

Shinozuka, M., Feng, M. Q., Kim, H., Uzawa, T., and Ueda, T. (2000). "Statistical analysis of fragility curves." Tech. Rep., Multidisciplinary Center for Earthquake Engineering Research, State University at Buffalo, Buffalo, N.Y.

\section{APPENDIX II. NOTATION}

The following symbols are used in this paper:

$a, a_{j}=$ PGA to which bridge $j$ is subjected;

$a_{y}, a_{p i}=$ spectral acceleration at yielding and $i$ th trial performance point on bilinear capacity spectrum, respectively;

$c=$ median of lognormal distribution;

$d_{l}=$ limit displacement defined as spectral displacement for specified state of damage;

$d_{y}, d_{p i}=$ spectral displacement at yielding and $i$ th trial performance point on bilinear capacity spectrum, respectively;

$F_{i}=$ laterally applied force on node $i$ for pushover analysis;

$F(\cdot)=$ fragility value for specified state of damage;

$f_{c}, f_{y}=$ ultimate strength of concrete and yield strength of reinforcing bar, respectively;

$g=$ acceleration due to gravity;

$H_{e}=$ length of elastic frame in finite-element model of column;

$i=$ node number;
$K=$ total number of bridges considered to develop fragility curve;

$L=$ likelihood function;

$L_{p}=$ length of plastic hinge, i.e., length of rigid frame in finite-element model of column;

$l=$ limit state of bridge;

$M=$ magnitude of ground-motion;

$m=$ mean of normal distribution;

$N=$ number of nodes in analysis model;

$P F=$ modal participation factor for fundamental mode;

$P_{j}\left(a, d_{l}\right)=$ probability that sample bridge $j$ experiences maximum spectral displacement greater than $d_{l}$ for time history group of $\mathrm{PGA}=a$;

$R=$ epicentral distance of ground-motion time history;

$S_{a}=$ spectral acceleration;

$S_{d}, S_{d}(a), \bar{S}_{d}(a)=$ spectral displacement, spectral displacement and mean spectral displacement for time-history group of $\mathrm{PGA}=a$, respectively;

$S R_{A}, S R_{V}=$ spectral reduction factors for range of constant peak spectral acceleration and constant peak spectral velocity of spectrum, respectively;

$T=$ period of fundamental mode;

$V=$ base shear;

$W, w_{i}=$ overall dead weight and dead weight assigned to node $i$ of bridge, respectively;

$(X)_{\text {damage }}=$ value of $X$ at specified state of damage;

$x_{j}=1$ or 0 depending on whether or not bridge sustains state of damage under PGA $=a_{j}$;

$\alpha=$ modal mass coefficient of fundamental mode;

$\beta_{0}, \beta_{\text {eff }}=$ equivalent viscous damping associated with full hysteresis loop area of capacity spectrum and effective viscous damping including assumed 5\% structural viscous damping, respectively;

$\Delta_{\text {girder }}=$ horizontal displacement of girder;

$\delta_{i}=$ lateral displacement at node $i$ due to lateral forces;

$\Phi[\cdot]=$ standardized normal distribution function;

$\phi_{\text {girder }}, \phi_{i}, \phi_{\mathrm{pl}}=$ amplitudes of fundamental mode at girder, node $i$, and plastic hinge, respectively;

$\kappa=$ damping modification factor;

$\sigma=$ standard deviation of normal distribution;

$\sigma_{d}(a)=$ standard deviation of spectral displacement for time-history group of $\mathrm{PGA}=a$;

$\sigma_{d}^{+}(a), \sigma_{d}^{-}(a)=+1 \sigma$ and $-1 \sigma \mathrm{SD}$ of spectral displacement for time-history group of $\mathrm{PGA}=a$, respectively;

$\theta_{\mathrm{p} 1}=$ rotation of plastic hinge; and

$\zeta=\log$-standard deviation of lognormal distribution. 